\title{
Total quality management (tqm) and systems thinking in translational science
}

\author{
Robert E Smith* \\ Park University, Parkville, MO 64152, USA
}

\begin{abstract}
Total Quality Management (TQM) and systems thinking are being used by pharmaceutical companies, physicians, nurses, researchers, academia and even patients to translate scientific discoveries into medical applications. They are collaborating openly and sharing research data, while they listen to the voices of the patients and their caregivers. Throughout the process of new drug development and patient care, systems-based thinking is useful. Moreover, the human body naturally follows the principles of TQM and systems thinking to maintain its health. In industry, TQM recognizes the importance of all the associates that are involved in a production process, regardless of their size or how much they are paid. Similarly, everything, from the smallest virus, to Bacteria, fungi, intestinal worms and the human eukaryotic cells that have 23 pairs of chromosomes are important in human health. The principles of TQM and systems-based thinking are described in this review article. These principles, together with important data indicate that people who have cancer and are starting radio- and/or chemotherapy should probably avoid taking dietary supplements that contain high doses of resveratrol, EGCG or other dietary phenolic compounds that activate the Nrf2/ARE signaling system. That is, this system is overactivated in multidrug resistant cancer. However, the prescription drug metformin (Glucophage ${ }^{\circledR}$ ) inhibits this system and could help save the lives of such patients, just as it saves the lives of patients who have type-2 diabetes.
\end{abstract}

\section{Introduction}

Total Quality Management (TQM) and systems thinking are being used in medicine and new drug development [1,2]. Systems thinking has become an important paradigm in many areas of science. Fritjof Capra described how it is important in modern physics and biology $[3,4]$. He and Pier Luigi Luisi further showed how it is important in mathematics, biology and medicine [5]. In systems thinking, the whole is greater than the sum of its parts [1-5]. Similarly, in TQM, team members and stake holders listen to the voice of the customers (or the patients) and interact synergistically to meet or exceed their expectations. This includes recognizing a deep ecology, in which humans are viewed as just one of many equally important parts of the global ecosystem [4,5]. Likewise, TQM recognizes the importance of all the associates that are involved in a production process, regardless of their job description or how much they are paid. Moreover, many patients and other members of society are calling for an end to animal testing. This is important for more than moral or ethical reasons. The results from animal testing are usually not applicable to humans. So, systems thinking teaches us to realize that the effects of prescription drugs depend on the system (or organism) to which they are given. What happens in one system (like a rodent) are often quite different than what happens in another (a human).

TQM is consistent with systems thinking $[1,2]$. This has led to a new code of behavior, in which data are shared, with an emphasis on quality rather the quantity of data. So, pharmaceutical companies and academic researchers that used to compete and keep their preclinical data secret are now sharing data. The results are a highly productive synergy. TQM and systems thinking also led to the development of predictive toxicology. That is, instead of testing a new molecular entity or drug on an animal, it can be tested on cells that come from the patient and are made from their own induced pluripotent stem cells (iPSCs). The former paradigm of using animals to evaluate the toxicity and efficacy of new drugs is no longer valid. Results from animal tests are seldom applicable to humans $[1,2]$.

One of the goals of this article is to describe how the tools and methods of systems thinking are being used to improve medical science and accelerate new drug development. This contrasts with the ways that medicine and new drug development were done previously using reductionist thinking. That is, many prescription drugs were developed based on their abilities to either activate or inhibit a single therapeutic target, such as an enzyme or an ion pump [1]. This was based on reductionist thinking that preached that one should look for the single root cause of a disease. More recently, many new drug candidates and medical devices are being developed based on their ability to modify the activities of many therapeutic targets $[1,2]$. This is based on systems thinking, which recognizes that complex problems (like diseases) have many causes. Not all of them are obvious upon initial inspection. So, researchers and pharmaceutical companies are looking for hidden connections, which is another important feature of systems thinking $[1,2,6]$. This includes looking for ways to affect protein-protein and protein-DNA interactions and the development of allo-network drugs that don't bind to an enzyme's active site [1]. This contrasts with reductionist thinking, in which a drug should bind to the active site of an enzyme or the ligand-binding site on a protein receptor or ion pump. This is how medicinal chemistry was once taught. However, there is a growing consensus that systems thinking is improving healthcare and new drug development dramatically $[1,2,7]$.

Correspondence to: Robert E. Smith, Science Department, Park University, 8700 NW River Park Drive, Parkville, MO 64152, USA, E-mail: Robert.smith05@ park.edu

Key words: TQM, systems thinking, translational science, deep ecology, animal testing, Nrf2/ARE, metformin, EGCG, resveratrol

Received: April 23, 2018; Accepted: April 28, 2018; Published: April 30, 2018 
Properly organized networks are essential for life and help make us human $[1,2,5]$. We are complex, self-regenerating, autopoietic organisms, whose bodies are ecosystems living in larger ecosystems. Life is sustained by a network of production processes, in which the function of almost every component is to participate in the production or transformation of itself and the other components in the network. So, network theory and systems thinking have emerged as guiding principles in biology and medicine $[1,2,5]$. A social, medical and scientific network has emerged from systems thinking. It enables predictive, preventive, personalized and participatory (P4) medicine [2].

We now treat the person and not just his or her disease $[1,2,5]$. We also realize that we all need some personalized treatments, even though some preventive treatments (like vaccines) are useful to almost everybody. All people (including homozygotic twins) are unique. Medical science is becoming much better at predicting every individual's susceptibility to different diseases by analyzing his or her genetics, epigenetics and metabolism. In addition, proper diet, physical activity and positive thinking, as well as avoiding unhealthy habits can help prevent diseases. When illnesses do emerge, patients and their caregivers can participate with healthcare professionals to come up with the best treatments and cures. All of this is compatible with the very natural process of TQM, which has been adopted throughout modern industry and which has always been a hallmark of good health and wellness. It is an essential part of systems thinking. Moreover, systems thinking is needed for proper TQM. Note that the term total quality leadership (TQL) is often used synonymously with TQM [1].

Systems thinking has been integrated into TQM for many years $[1,8]$. It is essential for survival in an increasingly competitive environment that requires efficient use of modern technologies, such as $3 \mathrm{D}$ and $4 \mathrm{D}$ printing. The health and productivity of an organization requires using a holistic approach. Traditional operating methods must be continuously evaluated and replaced when they become dysfunctional or unnecessary, just like ancestors of cetaceans (whales and dolphins) did when they no longer needed legs as they evolved into creatures that spent their entire lives in water $[1,8]$.

Even though it has not been recognized by most people, the same principles of TQM are used by our bodies to maintain good health and to prevent diseases [1]. Just as businesses listen to the voices of customers and communicate with them, cells within our bodies communicate with each other. Many medicinal chemists now realize that the human body is an ecosystem with a deep ecology. Human eukaryotic cells are just one component of the ecosystem. This is similar to the concept in TQM in which all associates are important to the organization. In modern biology, we are changing the way we think about what it is to be human. That is, the viruses, Bacteria, Archaea and other microorganisms in us help make us healthy humans. As in TQM, there is an extensive communication network among different microbes and between microbes and human (eukaryotic) cells. However, TQM in industry is often involved in manufacturing tools and machines. They don't make themselves. They are made by people. In contrast, living systems are not machines. We make ourselves in a process called autopoiesis (self-making). We make ourselves and we team with other people and organizations made by humans to improve all aspects of our healthcare. So, in biology, TQM means Total Quality Making, not Total Quality Manufacturing [1].

The principles of TQM are used by an extensive networking system in many governments [1]. They form teams that are equivalent to TQ teams used by businesses. For example, the FDA's Center of
Drug Evaluation and Research (CDER) and the Center for Biologics Evaluation and Research (CBER) are developing a more systematic approach for incorporating the patient's voice into new drug development. The goal is to build on the series of public workshops that occurred under the current patient-focused drug development program. This is being used to produce guidance's to assess the burdens of diseases and the treatments that are most important to patients, as well as impact measures, clinical outcome assessments, and endpoints to inform drug development and regulatory decisions [1].

\section{Discussion}

Systems thinking, and TQM help us realize that the effects of many drugs, dietary supplements and foods depend on the systems that consume them [1]. In addition, many successful drugs that were developed based on their effects on a single therapeutic target are now known to affect many targets. For example, metformin (Glucophage ${ }^{\oplus}$ ) is one of the most important drugs that was originally approved to treat one disease (type-2 diabetes, T2D) but may be useful in treating others, and possibly even slowing down aging [9-15]. It is the drug that is most frequently prescribed for lowering blood glucose in the first-line treatment of patients who have T2D, which affects one in nine adults in the USA [15]. It can also help reduce the occurrence of the top five comorbidities of T2D: cardiovascular diseases, cancer, depression, dementia and frailty. However, the extent to which metformin does this depends on the phenotype of the patient [15]. Metformin is also quite useful in preventing T2D in people who are pre-diabetic [11]. It not only reduces the concentration of glucose in the blood, but also offers protection against cardiovascular diseases [9]. Metformin also decreases the concentration of insulin, decreases IGF-1 signaling and inhibits mTOR (mammalian target of rapamycin) [11]. It reduces the incidence of cancer and mortality and helps people retain proper cognitive function. It does this by inhibiting the mitochondrial complex 1 in the electron transport chain, thus reducing the endogenous production of reactive oxygen species (ROS) [11]. At the same time, it targets histone acetylation in cancer-prone epithelial cells [13]. It may also affect aging by activating AMP-activated protein kinase (AMPK) and reducing DNA damage. So, metformin favorably influences metabolic and cellular processes that are closely linked to the development of age-related problems, such as inflammation, autophagy, and cellular senescence [11]. Since it is so inexpensive and easy to obtain, metformin could be especially useful in countries where many people don't have much money [9].

Perhaps the most exciting potential use of metformin (Glucophage $^{\oplus}$ ) is in extending the lifespan of not just people who have diabetes, but also in slowing down the aging process [9-15]. It does this by affecting several biochemical pathways that are important in how prediabetics age [10]. This includes increasing the concentrations of the following proteins while activating genes that code for them: $\mathrm{N}$-glycans (non-invasive surrogate markers of aging), SIRT1, p53 and p66Shc (an adaptor protein in the member of the SHC, or sarcoma homology family that functions as a redox enzyme that is linked to apoptosis). Metformin also increases the activity of SIRT1 and AMPK, while increasing the accessibility of the SIRT1 promoter, the length of telomeres and the accessibility of the SIRT1 promoter on chromatin [10]. It also decreases IGF-1 signaling, inhibits mTOR and the mitochondrial complex 1 in the electron transport chain, while reducing the production of ROS and reducing DNA damage [11]. Metformin also favorably influences metabolic and cellular processes that are linked to the development of age-related conditions, such as inflammation, autophagy, and cellular senescence [11]. 
Metformin (850 mg twice daily) was administered to subjects in a Diabetes Prevention Program (DPP) [11]. It also reduced the incidence of T2D by $31 \%$ compared to placebo after three years and prevented diabetes, as defined by HbA1C concentrations. Moreover, metformin improved risk factors for cardiovascular diseases and subclinical atherosclerosis (coronary artery calcium) in male participants [11].

Partly due to these important health benefits of metformin, some scientists are rejecting the idea that we die of old age [14]. Instead, we die of cumulative failures that occur within our cells and tissues. These failures are not inevitable breakdowns, are reversible conditions of aging. One of the most important of these is AMPK. Its concentration is relatively high when we are young but decreases with age. Recent studies have suggested that increasing the activity of AMPK can prevent and possibly reverse the life-shortening effects of aging [14]. Some scientists are beginning to refer to AMPK as a suppressor of aging itself $[14,16]$. It has been called a "metabolic master switch" [14,17]. It monitors the energy status of every human eukaryotic cell within us and triggers responses that maintain it within a healthy range [14]. That is, too little available energy starves the cell, while too much energy can produce too many reactive oxygen species (ROS) and disrupt cellular components. As AMPK decreases when we age, we become less energetic and more obese, while becoming increasingly vulnerable to cancer and diseases associated with impaired DNA and protein function. As a person accumulates abdominal fat, this leads to reduced insulin sensitivity, system-wide smoldering inflammation and metabolic syndrome. This, in turn, can lead to many forms of cancer, as well as cardiovascular, neurodegenerative and autoimmune diseases (including T2D). The modern Western lifestyle with its overabundance of nutrients and low level of physical activity exacerbates this. When a person's caloric intake is too high and/or physical activity too low, AMPK activation decreases. As a result, cells decrease their energyreleasing ATP-generating activities and shift to energy-storing processes that generate new fat deposits and make excess new glucose. Moreover, energy inefficiency eventually leads to the dysfunctions that are often described as inevitable diseases (or symptoms) of aging. So, restoring the activity of AMPK in the elderly may not only increase longevity, but also help to fight the symptoms of aging [14]. This hypothesis was supported by a clinical study in which subjects with T2D either received metformin (which activates AMPK) or placebo [16]. They were compared to subjects who did not have T2D. It was found that the subjects who were diabetic and received metformin lived a median of $\mathbf{1 5 \%}$ longer than did matched controls without diabetes [16]. So, there is a clinical trial underway, called Metformin in Longevity Study (MILES) [18] and another that is being planned called Targeting Aging with Metformin, or TAME, that will look at the effects of metformin on longevity [12].

However, there are some unexpected and hidden connections. Dietary antioxidants can help prevent smoldering inflammation and the diseases that it can cause. This includes cardiovascular, neurodegenerative and autoimmune diseases (such as T2D) as well as many types of cancer [1]. Phenolic compounds such as resveratrol and epigallocatechin gallate (EGCG) act as antioxidants by activating the nuclear erythroid-2 like factor-2 and antioxidant response elements Nrf2/ARE that exist in cells. However, metformin inhibits this system, instead of activating it. In some people, it exerts is many health effects despite this. However, in multidrug resistant cancer cells and the heart, the Nrf2/ARE system can have deadly effects when it is overactivated [19-24]. That is, the effects of an activated Nrf2/ARE system depend on context, or where it occurs, and on the degree to which it is activated
[19]. Its effects depend on the stage of the cancer, or the extent to which it has developed. Fully malignant cells are autonomous. They are very different from dysplastic (but not yet fully neoplastic) cells in a premalignant lesion. Premalignant cells are under much greater control from inflammatory cells and other stromal cells in their microenvironment. Moreover, they don't yet have enough DNA damage to make them autonomous. So, increasing Nrf2 activity, which would decrease both inflammatory and further oxidative or mutagenic stress, appears to be beneficial during premalignant states and may help limit further carcinogenesis. So, higher Nrf2 activity can be anticarcinogenic in the early stages of tumorigenesis, when the human body is still trying to control premalignant carcinogenesis. However, is can become pro-carcinogenic when it makes fully malignant cancer cells become resistant to treatment [19]. The prognosis of patients with tumors that have very an active Nrf2/ARE system is poor because it increases cancer cell proliferation and promotes chemoresistance and radioresistance [20]. Also, Nrf2 regulates the expression of the multidrug resistant protein-3 (MRP3) in both human bronchial epithelial and non-small cell lung cancer (NSCLC). This protein, when combined with upregulated detoxification enzymes like glutathione S-transferases (GSTs), can lead to the increased hydrophilicity of the cell membrane. This makes it easier for the cancer cells to excrete many anticancer drugs, including chlorambucil, cisplatin, etoposide, and doxorubicin [20].

Nrf2 also reprograms metabolic pathways towards anabolism, while augmenting purine synthesis and influencing the pentose phosphate pathway [21]. That is, Nrf2 redirects glucose and glutamine into anabolic pathways. Under physiological conditions, Nrf2 signaling is turned on by the presence of oxidative stressors in the cellular microenvironment, but is rapidly deactivated. However, under pathological conditions the tight regulation of Nrf2 changes. There is less responsiveness to cell stressors. At the same time, when Nrf2 is over-activated mammalian cells under adverse conditions have a survival advantage. This applies to cancers of liver, lung, colorectal, pancreas, prostate, gall bladder and ovaries [21].

So, metformin could save the lives of patients who are starting chemotherapy for cancer by preventing the Nrf2/ARE system from becoming overactivated. This has not been demonstrated in clinical trials, but it is quite possible. It's also quite possible that when pure resveratrol or EGCG are consumed at high doses in dietary supplements could activate the Nrf2/ARE system so much that it would prevent metformin from saving the lives of cancer patients who take them. This means that it would be wise for the investigators who are conducting the MILE trial or will conduct the SMILE trial to control or prevent the consumption of resveratrol, EGCG and many other supplements that contain high doses of nearly pure antioxidants. A partial list of dietary phenolic compounds that activate the Nrf2/ARE system can be found in a recent book [1].

\section{Conclusion}

The ways that TQM and systems thinking are being used in translational medicine were reviewed. That is, pharmaceutical companies, physicians, nurses, researchers, academia and even patients are working together to translate scientific discoveries into medical applications. They are collaborating openly and sharing research data, while they listen to the voices of the patients and their caregivers. Moreover, the human body naturally follows the principles of TQM to maintain its health. In industry, TQM recognizes the importance of all the associates that are involved in a production process, regardless of 
their position or how much they are paid. Similarly, everything, from the smallest virus, to Bacteria, fungi, intestinal worms and the human eukaryotic cells that have 23 pairs of chromosomes are important in human health and autopoiesis, or self-making. Systems thinking, and TQM also teach us that the effects of a prescription drug, dietary supplement or food depend on the system (or person) who takes them or eats them. For example, dietary phenolic compounds like resveratrol and EGCG may help prevent cancer by activating the Nrf2/ARE signaling system. However, once a patient is diagnosed with cancer and begins radio- and or chemotherapy, high doses of these same compounds could help cancer cells become multidrug resistant. Also, metformin has been shown to save lives, despite the fact that it inhibits the Nrf2/ARE signaling system. So, clinical trials that are testing the ability of metformin to extend one's lifespan and slow down aging should remember this. Participants in these studies probably should tell the study director if they take dietary supplements containing dietary phenolic compounds. In addition, primary care physicians and oncologists should probably tell any patient that is starting radioor chemotherapy to avoid taking such supplements. This could help the therapy be effective and prevent their cancer cells from becoming multidrug resistant.

\section{Acknowledgement}

Copy right: Robert E. Smith owns the copyright to this article.

\section{References}

1. Smith RE (2018) Systems Thinking in Medicine and New Drug Discovery, Volume One (Newcastle upon Tyne, Cambridge Scholars Publishing).

2. Smith RE (2018) Systems Thinking in Medicine and New Drug Discovery, Volume Two (Newcastle upon Tyne, Cambridge Scholars Publishing).

3. Capra F (1975) The Tao of Physics (Boulder, CO, Shambala Publications).

4. Capra F (1997) New York, The Web of Life. Anchor Books.

5. Capra F, Luisi PL (2014). A Systems View of Life: A Unifying Vision. (Cambridge, UK, Cambridge University Press).

6. Capra F (2002) The Hidden Connections. (New York, Doubleday).

7. Csermely P, Korcsmaros T, Kiss HJM, London G, Nussinov R, et al. (2013) Structure and dynamics of molecular networks: A novel paradigm of drug discovery. A comprehensive review. Pharmacol Therapeut 138: 333-408.
8. Jambekar AB (1994) Frameworks for integration of systems thinking with the total quality management practices. Conference Proceedings The 1994 International Systems Dynamics, Sterling, Scotland.

9. Bromage DI, Yellon DM (2015) The pleiotropic effects of metformin: time for prospective studies. Metformin clinical trial to increase longevity - pleiotropic effects. Cardiovasc Diabetol 14: 109.

10. De Kreutzenberg SV, Ceolotto G, Cattelan A, et al. (2015) Metformin improves putative longevity effectors in peripheral mononuclear cells from subjects with prediabetes. A randomized controlled trial. Nutr Metab Cardiovasc Dis 25: 686-693.

11. Wu L, Zhou B, Oshiro-Rapley N, et al. (2016) An ancient, unified mechanism for metformin growth inhibition in C. elegans and cancer. Cell 167: 1705-1718.

12. Barzilai N, Crandall JP, Kritchevsky SB, Espeland MA (2016) Metformin as a tool to target aging. Cell Metab 23: 1060-1065.

13. Cuyas E, Fernandez-Arroyo S, Joven J, Menendez JA (2016) Metformin targets histone acetylation in cancer-prone epithelial cells. Cell Cycle 15: 3355-3361.

14. Linton R (2015) AMPK and aging. Life Extension Magazine http://www.lifeextension. com/magazine/2015/11/ampk-and-aging/page-01

15. Wang C-P, Lorenzo C, Habib SL, et al. (2017) Differential effects of metformin on age related comorbidities in older men with type 2 diabetes. $J$ Diabetes Complications 31 : 679-686.

16. Clark WR (2002) A Means to an End: The Biological Basis of Aging and Health. (Oxford, UK, Oxford University Press).

17. Winder WW, Hardie DG (1999) AMP-activated protein kinase, a metabolic master switch: possible roles in type 2 diabetes. Am J Physiol 277: E1-10. U.S. National Institutes of Health. Metformin in Longevity Study (MILES). ClinicalTirals.gov (2017) https://clinicaltrials.gov/ct2/show/NCT02432287.

18. Sporn MB, Liby KT (2012) NRF2 and cancer: the good, the bad and the importance of context. Nat Rev Cancer 12: 1-9

19. Pandey P. Singh AK, Singh M, Tewari M, Shukla HS, et al. (2017) The seesaw of Keap1 Nrf2 pathway in cancer. Crit Rev Oncol Hematol 116: 86-98.

20. Menegon S, Columbano A, Giordano S (2016) The dual roles of NRF2 in cancer. Trend Mol Med 22: 578-593.

21. Chio IIC, Jafarnejad SM, Ponz-Sarvise M, Park Y, Rivera K, et al. (2016) NRF2 promotes tumor maintenance by modulating mRNA translation in pancreatic cancer. Cell 166: 963-976.

22. Bao L, Wu J, Dodson M, de la Vega EMR, Ning Y, et al. (2017) ABCF2, an Nrf2 target gene, contributes to cisplatin resistance in ovarian cancer cells. Mol Carcinogen 56: 1543-1553.

23. Leinonen HM, Kansanen E, Polonen P, Heinaniemi M, Levonen A-L, et al. (2015) Dysregulation of the Keap1-Nrf2 pathway in cancer. Biochem Soc Trans 43: 645-649.

Copyright: (C2018 Smith RE. This is an open-access article distributed under the terms of the Creative Commons Attribution License, which permits unrestricted use, distribution, and reproduction in any medium, provided the original author and source are credited. 